\title{
Erratum to: A Calculation Model of Beam Angular Divergence for EAST Neutral Beam Injector
}

\author{
Yongjian $\mathrm{Xu} \cdot$ Chundong $\mathrm{Hu} \cdot$ Yuanlai Xie • \\ Lizhen Liang $\cdot$ Jun Li $\cdot$ Ling Yu
}

Published online: 16 November 2010

(C) Springer Science+Business Media, LLC 2010

\section{Erratum to: J Fusion Energ}

\section{DOI 10.1007/s10894-010-9352-5}

In the original version of this article unfortunately the Eq. 9 had been erroneously published as:

$$
\begin{aligned}
i(x, y, z) & =\frac{I}{64 x_{0} y_{0}}\left[\operatorname{erf}\left(\frac{x_{0}-x}{a_{x}}\right)+\operatorname{erf}\left(\frac{x_{0}+x}{a_{x}}\right)\right] \cdot\left\{\operatorname{erf}\left(\frac{y_{0}-[y-(f-z) \tan B]}{a_{y}}\right)+\operatorname{erf}\left(\frac{y_{0}+[y+(f-z) \tan B]}{a_{y}}\right)\right. \\
& +\operatorname{erf}\left(\frac{y_{0}-[y-(f-z) \tan B]}{a_{y}}\right)+\operatorname{erf}\left(\frac{y_{0}+[y+(f-z) \tan B]}{a_{y}}\right)+\operatorname{erf}\left(\frac{y_{0}-[y-(f-z) \tan \theta]}{a_{y}}\right) \\
& \left.+\operatorname{erf}\left(\frac{y_{0}+[y+(f-z) \tan \theta]}{a_{y}}\right)+\operatorname{erf}\left(\frac{y_{0}-[y+(f-z) \tan \theta]}{a_{y}}\right)+\operatorname{erf}\left(\frac{y_{0}+[y+(f-z) \tan \theta]}{a_{y}}\right)\right\}
\end{aligned}
$$

And the correct equation is as follows:

$$
\begin{aligned}
i(x, y, z) & =\frac{I}{64 x_{0} y_{0}}\left[\operatorname{erf}\left(\frac{x_{0}-x}{a_{x}}\right)+\operatorname{erf}\left(\frac{x_{0}+x}{a_{x}}\right)\right] \cdot\left\{\operatorname{erf}\left(\frac{y_{0}-[y-(f-z) \tan B]}{a_{y}}\right)+\operatorname{erf}\left(\frac{y_{0}+[y-(f-z) \tan B]}{a_{y}}\right)\right. \\
& +\operatorname{erf}\left(\frac{y_{0}-[y+(f-z) \tan B]}{a_{y}}\right)+\operatorname{erf}\left(\frac{y_{0}+[y+(f-z) \tan B]}{a_{y}}\right)+\operatorname{erf}\left(\frac{y_{0}-[y-(f-z) \tan \theta]}{a_{y}}\right) \\
& \left.+\operatorname{erf}\left(\frac{y_{0}+[y-(f-z) \tan \theta]}{a_{y}}\right)+\operatorname{erf}\left(\frac{y_{0}-[y+(f-z) \tan \theta]}{a_{y}}\right)+\operatorname{erf}\left(\frac{y_{0}+[y+(f-z) \tan \theta]}{a_{y}}\right)\right\}
\end{aligned}
$$

The online version of the original article can be found under doi:10.1007/s10894-010-9352-5. 